\title{
CONTRACTING SPACELIKE HYPERSURFACES BY THEIR INVERSE MEAN CURVATURE
}

\author{
MICHAEL HOLDER
}

(Received 1 August 1998; revised 19 August 1999)

Communicated by K. Ecker

\begin{abstract}
In this work we study the behaviour of compact, smooth, orientable, spacelike hypersurfaces without boundary, which are immersed in cosmological spacetimes and move under the inverse mean curvature flow. We prove longtime existence and regularity of a solution to the corresponding nonlinear parabolic system of partial differential equations.
\end{abstract}

2000 Mathematics subject classification: primary 53C42, 53C50, 83C 30.

\section{Introduction}

A cosmological spacetime is a time-oriented connected Lorentzian manifold $\mathscr{V}$ containing a global Cauchy surface, thus we can write $\mathscr{V}=\Sigma^{n} \times I$ with $\Sigma^{n}$ being a Cauchy surface. In [8] Gerhardt obtained general existence and regularity results for prescribed mean curvature surfaces in cosmological spacetimes (in [1] Bartnik settled the corresponding problem for asymptotically flat spacetimes). In particular it was proved that in cosmological spacetimes which satisfy the timelike convergence condition and admit a big bang and a big crunch (this means suitable barriers in the future and past are known) there exists a foliation of $\mathscr{V}$ by hypersurfaces of constant mean curvature. Ecker and Huisken [6] studied the corresponding mean curvature flow and constructed hypersurfaces of prescribed mean curvature $\mathscr{H}$ in cosmological spacetimes satisfying the timelike convergence condition as an asymptotic limit of the geometric evolution equation $d / d s F=(H-\mathscr{H}) \nu$. So they gave an alternative proof for the existence of maximal and constant mean curvature surfaces. However they needed as well the assumption of future and past barriers.

(C) 2000 Australian Mathematical Society 0263-6115/2000\$A2.00+0.00 
In this paper we want to construct a foliation of the past of a spacelike hypersurface with strict positive mean curvature in a cosmological spacetime. For that purpose we look at the geometric evolution equation which deforms a given hypersurface in direction of its past directed unit normal where the speed at each point is determined by the inverse of the mean curvature at this point. The solution of the corresponding fully nonlinear parabolic system gives a foliation of the past of a spacelike hypersurface with strict positive mean curvature since the positivity of the mean curvature is preserved. To be more precise let $M_{0}$ be a smooth, orientable, compact, spacelike hypersurface without boundary in a cosmological spacetime $\mathscr{V}$ with strictly positive mean curvature given by an immersion

$$
F_{0}: M^{n} \rightarrow \mathscr{V}
$$

Then we solve the evolution equation

$$
\begin{aligned}
\frac{d}{d s} F(p, s) & =-\left(\frac{1}{H} v\right)(p, s), \quad p \in M^{n}, \quad s \geq 0, \\
F(p, 0) & =F_{0}(p) .
\end{aligned}
$$

where $v(p, s)$ denotes the future directed unit normal to $M_{s}:=F(\cdot, s)\left(M^{n}\right)$ at $F(p, s)$ and $H>0$ is the mean curvature on $M_{s}$.

Since $\mathscr{V}$ is a cosmological spacetime a spacelike hypersurface can be written as the graph of a real valued function $u$ over some fixed Cauchy surface $\Sigma^{n}$ and the fully nonlinear parabolic system (1) can be rewritten as a fully nonlinear equation for $u$. To derive longtime existence for (1) it is therefore necessary to develop a priori estimates on the height, the slope and the curvature. The a priori estimate for the slope is proved with methods related to that in [1] and [6], while the one for the curvature is proved by using a maximum principle for bilinear forms due to Hamilton [10].

This gives existence and therefore a foliation as long as the flow stays in a smooth compact region of the cosmological spacetime $\mathscr{V}$. Existence for all time follows then from an a priori estimate on the height $u$. To prove such an a priori estimate we make a natural regularity assumption (Section 5, Equation (5)) on the reference slicing, which is for example always satisfied in a Friedmann model (see the last section).

The evolution of hypersurfaces by mean curvature has been extensively studied in Riemannian ambient spaces. In [14,15] Huisken looked at the so-called mean curvature flow: $d / d s F=-H v$. This flow contracts compact initial surfaces and develops singularities as the flow decreases area (like the flow in the equation above). In the case of spacelike hypersurfaces in Lorentzian manifolds the mean curvature flow [6] increases rather than decreases area which leads to a more regular behaviour.

The flow along the inverse of the mean curvature in a Riemannian manifold plays an important role in the proof of the Penrose Inequality [17]. For this flow Gerhardt 
[9] and Urbas [23] proved independently longtime existence and convergence to an expanding round sphere if the initial surface is a compact star-shaped $C^{2, \alpha}$ hypersurface in $\mathbb{R}^{n}$.

After fixing notation and recalling some fundamentals in Section 2, we calculate the relevant evolution equations in Section 3 before we obtain the crucial a priori estimates in Section 4. The main results, longtime existence and existence for all time including the necessary height bound, are then proved in Section 5. In Section 6 we consider the case where the cosmological spacetime is a Robertson-Walker spacetime. We show, in particular, that assumption above is always satisfied in the Friedman model, a model for 'dust'.

The author wishes to thank Gerhard Huisken for his advice and encouragement while this work was undertaken.

\section{Preliminaries}

We consider $\mathscr{V}$ to be an $(n+1)$-dimensional smooth spacetime with a Lorentzian metric $\bar{g}=\left\{\bar{g}_{\alpha \beta}\right\}$ with signature $(-,+,+, \ldots,+)$. The canonical connection is denoted by $\bar{\nabla}$, the metric pairing by $\langle\cdot, \cdot\rangle$ and the curvature tensor by $\bar{R} m=\left\{\bar{R}_{\alpha \beta \gamma \delta}\right\}$. Greek indices range from 0 to $n$. As in [1] we shall assume the existence of a global time function $t \in C^{\infty}(\mathscr{V})$ with nonzero, past-directed timelike vector field $\bar{\nabla} t$. The reference slices $\mathscr{S}_{\mathrm{t}}:=\{p \in \mathscr{V} \mid t(p)=\mathrm{t}\}$ have future-directed unit normal vector

$$
T=-\psi \bar{\nabla} t
$$

where the lapse function $\psi \in C^{\infty}(\mathscr{V})$ is defined by

$$
\psi^{-2}:=-\langle\bar{\nabla} t, \bar{\nabla} t\rangle
$$

An adapted orthonormal frame on $\mathscr{S}_{1}$ will be denoted by $e_{0}, e_{1}, \ldots, e_{n}$ such that $e_{0}=T$. This adapted orthonormal frame defines a positive-definite norm $\|\cdot\|$ on tensors on $\mathscr{V}$, see [1] for more details.

Let $M$ be a smooth spacelike hypersurface in $\mathscr{V}$, which is embedded by some map

$$
F: M^{n} \rightarrow \mathscr{V}
$$

Let $v$ be the future directed timelike unit normal and choose locally an adapted orthonormal frame $\tau_{0}, \tau_{1}, \ldots, \tau_{n}$ in $\mathscr{V}$ such that restricted to $M$ we have $\tau_{0}=\nu$. We will denote by $g=\left\{g_{i j}\right\}$ and $R m=\left\{R_{i j k l}\right\}$ the induced metric and the Riemann curvature tensor on $M$ respectively, where Latin indices run from 1 to $n$. The covariant derivative on $M$ will be denoted by $\nabla$ and the Laplace-Beltrami operator by $\Delta$. The second fundamental form $A=\left\{h_{i j}\right\}$ on $M$ is given by

$$
h_{i j}:=\left\langle\bar{\nabla}_{\tau_{i}} \nu, \tau_{j}\right\rangle=-\left\langle\nu, \bar{\nabla}_{\tau_{i}} \tau_{j}\right\rangle .
$$


As in $[6,14]$ we sum over repeated indices

$$
H=h_{i i}, \quad|A|^{2}=h_{i j} h_{i j} .
$$

The curvature, Ricci curvature Ric $=\left\{R_{i j}\right\}$ and the scalar curvature $R$ on $M$ are given by the Gauss' equation

$$
\begin{aligned}
R_{i j k l} & =\bar{R}_{i j k l}-h_{i k} h_{j l}+h_{i l} h_{j k}, \\
R_{i k} & =\bar{R}_{i k}-H h_{i k}+h_{i l} h_{l k}+\bar{R}_{i 0 k 0}, \\
R & =\bar{R}-H^{2}+|A|^{2}+2 \overline{\operatorname{Ric}}(v, v) .
\end{aligned}
$$

The Codazzi equations state that:

$$
\nabla_{k} h_{i j}-\nabla_{j} h_{i k}=\bar{R}_{0 i j k} .
$$

We have the following rule for changing covariant derivatives:

$$
\nabla_{i} \nabla_{j} Y_{k}-\nabla_{j} \nabla_{i} Y_{k}=-R_{i j k l} Y_{l}
$$

From these equations we can get the following fundamental identity, see for example $[3,20,22]$.

LEMMA 2.1. We have

$$
\begin{aligned}
\Delta h_{i j}= & \nabla_{i} \nabla_{j} H+\bar{\nabla}_{j} \bar{R}_{0 k i k}+\bar{\nabla}_{k} \bar{R}_{0 i j k}+H \bar{R}_{0 i j 0}+h_{i j}\left(\overline{\operatorname{Ric}}(\nu, v)+|A|^{2}\right) \\
& +h_{i l} \bar{R}_{l k j k}+h_{l j} \bar{R}_{l k i k}+2 h_{l k} \bar{R}_{l i j k}-H h_{i k} h_{k j},
\end{aligned}
$$

The a priori estimates are expressed in terms of the height function

$$
u(p):=t(F(p))
$$

and the gradient function $v$, which measures the angle between $M$ and the reference slices $\mathscr{S}_{\mathrm{t}}$, given as

$$
v:=-\langle\nu, T\rangle
$$

Like in $[1,(2.8)]$ we get the identity

$$
\Delta u=\operatorname{div} \bar{\nabla} t+\psi^{-1} v H .
$$

Furthermore, we have the inequality

$$
\left|\left\langle\tau_{\alpha}, e_{\beta}\right\rangle\right| \leq v
$$


for all $0 \leq \alpha, \beta \leq n$. This gives an estimate for the restriction of any $p$-tensor $B \in T^{p}(\mathscr{V})$ on $M$

$$
\left\|\left.B\right|_{T M}\right\| \leq v^{p}\|B\| .
$$

For a tensor $B$ we denote by $\|B\|_{l, G}$ the $C^{l}$-norm of $B$ in the domain $G \subset \mathscr{V}$.

For the convenience of the reader let us recall the first variation formula for $H$ (see $[1,4,7]$ ) with respect to a deformation of the surrounding space generated by an arbitrary transverse vector field $X$

$$
X\left(H_{X}\right)=-\Delta\langle X, v\rangle+\langle X, v\rangle\left(\left|A^{2}\right|+\overline{\operatorname{Ric}}(\nu, v)\right)\langle X, \nabla H\rangle,
$$

or in terms of the Killing tensor $\mathscr{L}_{T} g(X, Y)=\left\langle\bar{\nabla}_{X} T, Y\right\rangle+\left\langle\bar{\nabla}_{Y} T, X\right\rangle$,

$$
\begin{aligned}
T\left(H_{T}\right)= & \frac{1}{2}\left(\bar{\nabla}_{v} \mathscr{L}_{T} g\right)\left(e_{i}, e_{i}\right)-\left(\bar{\nabla}_{e_{i}} \mathscr{L}_{T} g\right)\left(v, e_{i}\right)-\frac{1}{2} H \mathscr{L}_{T} g(v, v) \\
& -\mathscr{L}_{T} g\left(e_{i}, e_{j}\right) A\left(e_{i}, e_{j}\right) .
\end{aligned}
$$

\section{Evolution equations}

Starting from the evolution equation

$$
\frac{d}{d s} F=-\frac{1}{H} \nu,
$$

we derive evolution equations for the height, the gradient, the curvature and other relevant quantities on $M_{s}$.

Proceeding exactly as in [14], we first compute the derivative of the metric $g$, volume element $\mu$ and the unit normal $\nu$ on $M$

LEMMA 3.1. We have the equations

$$
\begin{aligned}
& \frac{d}{d s} g_{i j}=-2 \frac{1}{H} h_{i j}, \\
& \frac{d}{d s} v=\nabla\left(-\frac{1}{H}\right), \\
& \frac{d}{d s} \mu=H\left(-\frac{1}{H}\right) \mu=-\mu .
\end{aligned}
$$

We now notice that

$$
\frac{d}{d s} u=-\frac{1}{H} \psi^{-1} v
$$


and

$$
\frac{d}{d s} v=-\left\langle T, \frac{1}{H^{2}} \nabla H\right\rangle+\frac{1}{H}\left\langle\nabla_{v} T, v\right\rangle,
$$

such that from (2) and (4) we obtain

\section{LEMMA 3.2.}

(i) $\left(\frac{d}{d s}-\frac{1}{H^{2}} \Delta\right) u=-\frac{1}{H^{2}} \operatorname{div} \bar{\nabla} t-\frac{2}{H} \psi^{-1} v$,

(ii) $\left(\frac{d}{d s}-\frac{1}{H^{2}} \Delta\right) v=\frac{1}{H}\left\langle\bar{\nabla}_{\nu} T, v\right\rangle-\frac{1}{H^{2}} T\left(H_{T}\right)-\frac{1}{H^{2}} v\left(\left|A^{2}\right|+\overline{\operatorname{Ric}}(\nu, v)\right)$.

Finally we derive the evolution equation for the second fundamental form.

\section{LEMMA 3.3. We have the equations}

(i)

$$
\begin{aligned}
\left(\frac{d}{d s}-\frac{1}{H^{2}} \Delta\right) h_{i j}= & -2 \frac{1}{H^{3}} \nabla_{i} H \nabla_{j} H-2 \frac{1}{H} \bar{R}_{i 00 j}-2 \frac{1}{H^{2}} h_{k l} \bar{R}_{l i j k} \\
& -\frac{1}{H^{2}} h_{j l} \bar{R}_{l k i k}-\frac{1}{H^{2}} h_{l i} \bar{R}_{l k j k}-\frac{1}{H^{2}} \bar{\nabla}_{k} \bar{R}_{0 i j k} \\
& -\frac{1}{H^{2}} \bar{\nabla}_{j} \bar{R}_{0 k i k}-\frac{1}{H^{2}} h_{i j}\left(\left|A^{2}\right|+\overline{\operatorname{Ric}}(v, v)\right) \\
\left(\frac{d}{d s}-\frac{1}{H^{2}} \Delta\right) H= & -2 \frac{1}{H^{3}} \nabla_{i} H \nabla_{i} H+\frac{1}{H}\left(\left|A^{2}\right|+\overline{\operatorname{Ric}}(v, v)\right)
\end{aligned}
$$

(ii)

PROOF. Like in [15] we get

$$
\frac{d}{d s} h_{i j}=\nabla_{i} \nabla_{j}\left(-\frac{1}{H}\right)-\frac{1}{H}\left(h_{i l} h_{l j}+\bar{R}_{i 00 j}\right)
$$

and with Lemma 2.1, (i) follows. The second identity is an immediate consequence of (4) and can also be derived by taking the trace of (i).

\section{A priori estimates}

In this and in the following section we assume that $\mathscr{V}$ is a cosmological spacetime, thus it is connected, globally hyperbolic and admits a compact Cauchy surface (thus $\mathscr{V}=\Sigma \times I)$. This implies, in particular, the existence of a global time function $t \in C^{\infty}(\mathscr{V})$ as in Section 2. Moreover, we assume that the timelike convergence condition is satisfied, thus

$$
\overline{\operatorname{Ric}}(X, X) \geq 0
$$


holds for all timelike vector fields $X$. The timelike convergence condition is only needed for the lower bound on the mean curvature.

Now we prove the a priori estimates, which are necessary for the proof of Theorem 5.1, the main result. With Lemma 4.1 we get a lower a priori bound on the mean curvature $H$ which implies an upper bound on the height function $u$. Then we get the crucial a priori bound on the gradient function $v$. To prove this estimate we proceed in the same way as Bartnik did in [1, Theorem 3.1]. Using the derived a priori estimates we get an upper a priori estimate on the mean curvature $H$ and, using a maximum principle for bilinear forms as Hamilton formulated it in [10], an a priori bound for the full second fundamental form. So we have, as long as the flow stays in a smooth region of $\mathscr{V}$ where $t$ is bounded, a a priori $C^{2}$ bound for the height function $u$ which combined with estimates due to Krylov yields to an a priori $C^{2, \alpha}$ bound of $u$ and thus to full regularity.

LEMMA 4.1. Let $g=g(t, y)$ be a smooth function with $t \in \mathbb{R}, y \in \mathbb{R}^{k}$. Let

$$
f(t)=\inf \{g(t, y): y \in Y\},
$$

where $Y$ is a compact set. Then $f(t)$ is Lipschitz and

$$
\frac{\partial}{\partial t} f(t) \geq \inf \left\{\frac{\partial}{\partial t} g(t, y): y \in Y(t)\right\},
$$

with $Y(t)=\{y: g(t, y)=f(t)\}$.

For a proof see [11].

From now on let $M_{s}$ be a smooth solution of (1) on the interval $0 \leq s<s_{0}$, such that $M_{s}$ is contained in a smooth compact subset $G \subset \mathscr{V}$ for all $0 \leq s<s_{0}$ and we assume that $H \geq H_{0}>0$ on $M_{0}$.

PROPOSITION 4.1. Since $H \geq H_{0}>0$ on $M_{0}$, we have the following a priori bound for the mean curvature on $M_{s}$

$$
H \geq e^{s / n} H_{0}
$$

Proof. We only need to show that the function $f(s):=\min \left\{H e^{-s / n}(p, s): p \in\right.$ $\left.M^{n}\right\}$ is growing, thus

$$
\frac{d}{d s} f \geq 0
$$

Define $Y(s):=\left\{p: H e^{-s / n}(p, s)=f(s)\right\}$ and use Lemma 4.1

$$
\frac{d}{d s} f(s) \geq \min \left\{\frac{d}{d s}\left(H e^{-s / n}\right)(p, s) \mid p \in Y(s)\right\}
$$




$$
\begin{aligned}
= & \min \left\{\left(e ^ { - s / n } \left(\frac{1}{H^{2}} \Delta H-\frac{2}{H^{3}} \nabla_{i} H \nabla_{i} H\right.\right.\right. \\
& \left.\left.\left.+\frac{1}{H}\left(|A|^{2}+\overline{\operatorname{Ric}}(\nu, \nu)-\frac{1}{n} H^{2}\right)\right)\right)(p, s) \mid p \in Y(s)\right\} \\
\geq & \min \left\{\left(e^{-s / n}\left(0-0+\frac{1}{n} H+0-\frac{1}{n} H\right)\right)\right\}=0 .
\end{aligned}
$$

REMARK 4.1. As a direct consequence to the above equation we have $u \leq \max _{M_{0}} u$, since $d / d s u=-1 / H \psi^{-1} v$.

PROPOSITION 4.2. The gradient function $v$ satisfies on $M_{s}$

$$
v(p, s) \leq\left(1+\sup _{M_{0}} v\right) \exp \left(\lambda\left[\max _{M_{0}} u-u(p, s)\right]\right) \exp (\hat{K} s)
$$

where

$$
\lambda=\lambda\left(n,\left\|\mathscr{L}_{T} g\right\|_{1, G},\|T\|_{1, G},\left\|\psi^{-1}\right\|_{1, G}\right), \quad \hat{K}=2 \frac{2 n}{4 n+1}+\frac{1}{2} \lambda .
$$

PROOF. Following the argument in [1, Theorem 3.1] let $\lambda, \hat{K}>0$ be constants chosen later and define

$$
C_{\lambda}:=\left(1+\sup _{M_{0}} v\right) e^{\lambda \max _{M_{0}} u}
$$

Suppose $v e^{\lambda u-\hat{K} s}$ reaches $C_{\lambda}$ for the first time at $\left(p_{1}, s_{1}\right) \in M^{n} \times\left(0, s_{0}\right]$. Then at this point

$$
\begin{aligned}
& 0 \leq\left(\frac{d}{d s}-\frac{1}{H^{2}} \Delta\right) v e^{\lambda u-\hat{K} s}, \\
& 0=\nabla\left(v e^{\lambda u-\hat{K} s}\right) .
\end{aligned}
$$

From Lemma 3.2 and [1, Proof of Theorem 3.1] we infer

$$
\begin{aligned}
\left(\frac{d}{d s}-\frac{1}{H^{2}} \Delta\right) u & \leq\left|\left(\frac{d}{d s}-\frac{1}{H^{2}} \Delta\right) u\right| \leq \frac{1}{H^{2}} C_{0} v^{2}+\frac{2}{H} \psi^{-1} v \\
& \leq \frac{1}{H^{2}} C_{1}\left(C_{0}\right) v^{2}+\frac{1}{2}
\end{aligned}
$$

as well as

$$
\left(\frac{d}{d s}-\frac{1}{H^{2}} \Delta\right) v \leq-\frac{1}{H^{2}} v|A|^{2}+\frac{1}{H^{2}} C_{2}\left(v^{3}+|A| v^{2}\right)
$$


where

$$
C_{0}=C_{0}\left(\left\|\psi^{-1}\right\|_{1, G}\right) \quad \text { and } \quad C_{2}=C_{2}\left(\left\|\mathscr{L}_{T} g\right\|_{1, G},\|T\|_{1, G}, n\right)
$$

Here we also use (3) and the timelike convergence condition. Hence we conclude

$$
\frac{1}{H^{2}} v|A|^{2} \leq \frac{1}{H^{2}} C_{2}|A| v^{2}+\frac{1}{H^{2}}\left(C_{2}+C_{0} \lambda\right) v^{3}+\frac{1}{2} v \lambda+\frac{1}{H^{2}} \lambda^{2}|\nabla u|^{2} v-\hat{K} v .
$$

Proceeding as in $[1$, Theorem 3.1$]$ we obtain

$$
v|A|^{2} \geq\left(1+\frac{1}{2 n}\right) \lambda^{2} v|\nabla u|^{2}-C_{3}\left(n,\|T\|_{1, G}\right) v^{3}-H^{2} v
$$

Set $\hat{K}=2(2 n) /(4 n+1)+\lambda / 2$ and since $|\nabla u|^{2}=\psi^{-2}\left(v^{2}-1\right)$ we finally arrive at

$$
\frac{1}{2 n} \lambda^{2} \psi^{-2}\left(v^{2}-1\right) \leq C_{4} v^{2}(\lambda+1) \text {. }
$$

at $\left(p_{1}, s_{1}\right)$ where $C_{4}$ depends on $C_{0}, C_{1}, C_{2}$ and $C_{3}$. Since by definition of $C_{\lambda}$ we have $v\left(p_{1}, s_{1}\right) \geq 2$ and we derive a contradiction for large enough $\lambda$.

PROPOSITION 4.3. We have the following a priori upper bound for the mean curvature $H$ on $M_{s}$

$$
H \leq C_{5},
$$

where

$$
C_{5}=\max \left\{\sup _{M_{0}} H v^{q}, \sup _{M^{n} \times\left[0, s_{0}\right]}\left(\frac{1}{H} C_{6}\right)\right\}
$$

with $q:=1+\varepsilon, C_{6}=C_{6}\left(n, \varepsilon^{-1},\left\|\mathscr{L}_{T} g\right\|_{1, G},\|T\|_{1, G}\right)$ and $\varepsilon>0$ arbitrary but fuxed.

Proof. Suppose $H v^{q}$ reaches $C_{5}$ for the first time, at $\left(p_{1}, s_{1}\right) \in M^{n} \times\left(0, s_{0}\right]$. Then at this point

$$
\begin{gathered}
\nabla\left(H v^{q}\right)=0, \\
\left(\frac{d}{d s}-\frac{1}{H^{2}} \Delta\right) H v^{q} \geq 0
\end{gathered}
$$

From Lemma 3.3 (ii) and the proof of Proposition 4.4 we have

$$
\left(\frac{d}{d s}-\frac{1}{H^{2}} \Delta\right) H=-2 \frac{1}{H^{3}} \nabla_{i} H \nabla_{i} H+\frac{1}{H}\left(\left|A^{2}\right|+\overline{\operatorname{Ric}}(\nu, v)\right)
$$


and

$$
\left(\frac{d}{d s}-\frac{1}{H^{2}} \Delta\right) v \leq-\frac{1}{H^{2}} v|A|^{2}+\frac{1}{H^{2}} C_{2}\left(v^{3}+|A| v^{2}\right) .
$$

Using again the fact that $\overline{\operatorname{Ric}}(\nu, \nu) \geq 0$ we conclude

$$
\begin{aligned}
0 & \leq \frac{1}{H} C_{2} v^{q+2}-(q-1) \frac{1}{H}|A|^{2} v^{q}+\frac{C_{2}}{H}|A| v^{q+1} \\
& \leq \frac{C_{2}}{H} v^{q+2}+\frac{C_{2}^{2}}{4 \tilde{\varepsilon} H} v^{q+2}-(q-1-\tilde{\varepsilon}) \frac{1}{H}|A|^{2} .
\end{aligned}
$$

Since $|A|^{2} \geq 1 / n H^{2}$ we finally get at the point $\left(p_{1}, s_{1}\right)$ by choosing $\tilde{\varepsilon}=\varepsilon / 2$

$$
H v^{q} \leq \frac{1}{H} C_{6} \leq C_{5} .
$$

The proposition follows because $H \leq H v^{q}$.

PROPOSITION 4.4. For the second fundamental form $h_{i j}$ satisfies the following a priori estimate on $M_{s}$

$$
h_{i j} \leq \bar{C}_{0} g_{i j},
$$

where

$$
\bar{C}_{0}=\max \left\{\sup _{M^{n} \times\left[0, s_{0}\right]}\left(\frac{1}{2}+2 H+4 \bar{C}_{1}\right), \sup _{M_{0}}|A|\right\},
$$

with $\bar{C}_{1}=\bar{C}_{1}\left(H_{0},\|\bar{R} m\|_{1, G}\right)$ and $H_{0}$ like in Proposition 4.2 .

REMARK 4.2. It's enough to show an upper bound on $h_{i j}$, because $H$ is strictly positive.

PROOF. We show that the eigenvalues of

$$
M_{i j}=\left(\bar{C}_{0} g_{i j}-h_{i j}\right)
$$

remain nonnegative. First of all we need an evolution equation for $M_{i j}$. Using Lemma 3.3 (i) and Lemma 3.1 (i) we get

$$
\left(\frac{d}{d s}-\frac{1}{H^{2}} \Delta\right) M_{i j}=N_{i j},
$$


where

$$
\begin{aligned}
N_{i j}= & -2 \bar{C}_{0} \frac{1}{H} h_{i j}+2 \frac{1}{H^{3}} \nabla_{i} H \nabla_{j} H+2 \frac{1}{H} \bar{R}_{i 00 j}+2 \frac{1}{H^{2}} h_{k l} \bar{R}_{l i j k}+\frac{1}{H^{2}} h_{j l} \bar{R}_{l k i k} \\
& +\frac{1}{H^{2}} h_{l i} \bar{R}_{l k j k}+\frac{1}{H^{2}} \bar{\nabla}_{k} \bar{R}_{0 i j k}+\frac{1}{H^{2}} \bar{\nabla}_{j} \bar{R}_{0 k i k}+\frac{1}{H^{2}} h_{i j}\left(\left|A^{2}\right|+\overline{\operatorname{Ric}}(\nu, \nu)\right) .
\end{aligned}
$$

In [10, Theorem 9.1] a maximum principle for such an evolution equation was proved under the assumption that the absolute term $N_{i j}$ is a polynomial of $M_{i j}$ and $g_{i j}$. Since $\bar{R} m$ is smooth and $H$ is bounded, it is easy to see that the argument is valid in our case as well. We have then only to consider the first time $s_{0}$, where at some point $p \in M_{s_{0}}$ a zero eigenvector $v=\left\{v_{i}\right\}$ of $M_{i j}$ occurs, and Proposition 4.6 is proved if we can show that $N_{i j} v^{i} v^{j}$ is non-negative. For that purpose we choose an orthonormal basis $\left(e_{1}, \ldots, e_{n}\right)$ for $T_{p} M_{s_{0}}$ such that $h_{i j}$ (and thus $M_{i j}$ ) becomes diagonal. Let us assume that $v=e_{1}$ and that $\lambda_{1}, \ldots, \lambda_{n}$ are the eigenvalues of $h_{i j}$ at $p$. Then from $M_{11}=0$ it follows that $\lambda_{1}=\bar{C}_{0}$ at $p$ and we obtain

$$
\begin{aligned}
N_{i j} v^{i} v^{j}=N_{11} & \geq-2 \bar{C}_{0} \frac{1}{H} \lambda_{1}+2 \frac{1}{H^{3}} \nabla_{e_{1}} H \nabla_{e_{1}} H-\frac{1}{2} \frac{1}{H^{2}}|A|^{2}-\frac{1}{H^{2}} \bar{C}_{1}+\frac{1}{H^{2}} \lambda_{1}|A|^{2} \\
& \geq-2 \bar{C}_{0}^{2} \frac{1}{H}-\frac{1}{H^{2}} \bar{C}_{1}+\frac{1}{H^{2}}\left(\bar{C}_{0}-\frac{1}{2}\right) \bar{C}_{0}^{2} \\
& \geq \frac{1}{H^{2}}\left(\bar{C}_{0}-\frac{1}{2}-2 H\right) \bar{C}_{0}^{2}-\frac{1}{H^{2}} \bar{C}_{1} \\
& \geq \frac{1}{H^{2}}\left(\bar{C}_{0}-\frac{1}{2}-2 H-4 \bar{C}_{1}\right) \bar{C}_{0}^{2} \geq 0 .
\end{aligned}
$$

It is well known that one can get $C^{0, \alpha}$ a priori estimates on $\nabla^{2} u$ in $G$ from the a priori estimates on $v, H$ and $h_{i j}$ (like in the work of Gerhardt [9, Theorem 4.2] and Urbas $[23,(3.55)])$.

PROPOSITION 4.5. The second derivatives of the height function $u$ are uniformly bounded in $C^{0, \alpha}(G)$.

For the proof we refer to [18, Chapter 5].

REMARK 4.3. With Proposition 4.5 and standard results from regularity theory, we get the full regularity of $u$.

\section{Longtime existence}

With the help of the a priori estimates derived in the last section, we now obtain the main longtime existence result. 
THEOREM 5.1. Let $\mathscr{V}=\Sigma^{n} \times I$ be a cosmological spacetime satisfying the timelike convergence condition and suppose that $M_{0}=F_{0}\left(M^{n}\right)$ is a smooth, compact, spacelike hypersurface in $\mathscr{V}$. Then there exists a unique family $M_{s}=F(\cdot, s)\left(M^{n}\right)$ of smooth compact spacelike hypersurfaces satisfying the initial value problem (1) on an interval $0 \leq s<s_{0}$. Moreover, if $M_{s}$ remains in a smooth region of $\mathscr{V}$ where $t$ is bounded as $s \rightarrow s_{0}$, then the solution can be extended beyond $s_{0}$.

PROOF. It is easy to see that for short times the nonlinear system (1) reduces to a nonlinear scalar equation in $u$ which is strictly parabolic (see [5]). Then, if the initial surface $M_{0}$ is smooth, short time existence of a unique smooth solution of (1) follows from standard parabolic theory, see for example [12]. Now let $s_{0}$ be the maximal time of existence. Since by assumption $M_{s}$ stays in a compact smooth region of $\mathscr{V}$ and therefore $u$ is uniformly bounded, we infer from the last section that $M_{s}$ converges to a smooth surface $M_{s_{0}}$ as $s \rightarrow s_{0}$. Thus the solution $M_{s}$ can be extended beyond $s_{0}$ by means of the local existence result.

LEMMA 5.1. If the 'inverse mean curvature flow' (1) exists for all times then the area of $\left(M_{s}\right)$ tends to zero as $s \rightarrow \infty$.

Proof. The volume decreases exponentially, see Lemma 4.1 (iii), $d / d s \int d \mu=$ $-\int d \mu$.

To prove existence for all times we need an a priori estimate on the height function $u$ which can only be derived by an regularity assumption on the reference slicing. For simplicity assume that the 'big bang' is at $t=0$.

DEFINITION 5.1. We will call a spacetime 'slicing-natural' if this spacetime satisfies

$$
\begin{gathered}
-\check{\delta} \frac{d}{d t} H_{\mathscr{S}_{1}} \leq\left(\left|A_{\mathscr{S}_{i}}\right|^{2}+\overline{\operatorname{Ric}}(T, T)\right) \leq-\check{C} \frac{d}{d t} H_{\mathscr{S}_{r}} \text { and } \\
\frac{\hat{\delta}}{H_{\mathscr{S}_{1}}} \leq t \leq \frac{\hat{C}}{H_{\mathscr{S}_{i}}} .
\end{gathered}
$$

Here $H_{\mathscr{S}_{1}},\left|A_{\mathscr{S}_{1}}\right|^{2}$ and $T$ are the mean curvature, total curvature and unit normal of the reference slices $\mathscr{S}_{t}$.

Note that the first equation ensures that the lapse function is bounded from above and below which could be alternatively assumed. Examples for 'slicing-natural' spacetimes are for example the Robertson Walker spacetimes which model dust or radiation, the Kasner cosmological model [MTW] and the Mixmaster like universe, a Kasner model with time depending exponents. The model spacetimes for dust, the so called Friedmann models will be discussed in the next section. If we already have a 
foliation by constant mean curvature surfaces then we can set: $t:=2 / H_{\mathscr{S}}$, so that (5) reduces to $\left(\left|A_{\mathscr{S}_{1}}\right|^{2}+\overline{\operatorname{Ric}}(T, T)\right) \leq \tilde{C} H_{\mathscr{S}_{1}}^{2}$.

PROPOSITION 5.1. Let $\mathscr{V}$ be a 'slicing-natural' spacetime, then the height function fulfills on $M_{s}$

$$
\exp (-C s) \inf _{M_{0}} u \leq u \leq \exp (-\delta s) \sup _{M_{0}} u
$$

PROOF. First we show that the lapse function $\psi$ is bounded from above and below under the assumption (5).

We have (constraint equations) $d / d t H_{\mathscr{S}_{t}}=\Delta \psi-\psi\left(\left|A_{\mathscr{S}_{1}}\right|^{2}+\overline{\operatorname{Ric}}(T, T)\right)$. So we have a point $(p, s) \in \mathscr{V}$ where $\psi$ attains its maximum

$$
\frac{d}{d t} H_{\mathscr{S}_{1}} \leq-\psi\left(\left|A_{\mathscr{S}_{1}}\right|^{2}+\overline{\operatorname{Ric}}(T, T)\right) \Longleftrightarrow \psi \leq \frac{-d\left(H_{\mathscr{S}_{1}}\right) / d t}{\left(\left|A_{\mathscr{S}_{1}}\right|^{2}+\overline{\operatorname{Ric}}(T, T)\right)} \leq \frac{1}{\check{\delta}} .
$$

Therefore, $\psi \leq 1 / \check{\delta}$ everywhere. At a point $(p, s) \in \mathscr{V}$ where $\psi$ attains its minimum we have

$$
\frac{d}{d t} H_{\mathscr{S}_{1}} \geq-\psi\left(\left|A_{\mathscr{S}_{1}}\right|^{2}+\overline{\operatorname{Ric}}(T, T)\right) \Longleftrightarrow \psi \geq \frac{-d\left(H_{\mathscr{S}_{t}}\right) / d t}{\left(\left|A_{\mathscr{S}_{t}}\right|^{2}+\overline{\operatorname{Ric}}(T, T)\right)} \geq \frac{1}{\check{C}}
$$

Therefore, $\psi \geq 1 / \check{C}$ everywhere.

Now we use the fact that $\Delta u \leq 0$ and $v=1$ in a point where $u$ attains its maximum. This gives us

$$
\begin{aligned}
0 & \geq \Delta u=\operatorname{div} \bar{\nabla} t+H \psi v=-\operatorname{div}\left(T \psi^{-1}\right)+H \psi^{-1} \\
& \geq-\underbrace{\left\langle\bar{\nabla}_{\tau_{i}} T, \tau_{i}\right\rangle}_{\left\langle\bar{\nabla}_{e_{i}} T_{e_{i}}\right\rangle=H_{S_{i}}} \psi^{-1}-\underbrace{\left\langle T, \tau_{i}\right\rangle}_{=0} \bar{\nabla}_{\tau_{i}} \psi^{-1}+H \psi^{-1} \text { if and only if } H \leq H_{\mathscr{S}_{i}} .
\end{aligned}
$$

In a point where $u$ attains its minimum we get in the same way $H \geq H_{\mathscr{S}_{1}}$.

For the lower bound of $u$ we show that the function $g(s):=\min _{M_{s}} u \exp (C s)$ is nondecreasing, thus $d / d s g \geq 0$. This follows directly from (5), $d / d s u=-1 / H \psi^{-1} v$ and the above equations. For the upper bound one shows in the same way that the function $\bar{g}:=\max _{M_{s}} u \exp (\delta s)$ is nonincreasing. Here $C=1 / \hat{\delta} \delta$ and $\delta=1 / \hat{C} \check{C}$ and are therefore only depending on the constants of (5).

THEOREM 5.2. In a 'slicing-natural' spacetime $\mathscr{V}$ we have that the 'inverse mean curvature flow' (1) exists for all times, the area of $\left(M_{s}\right)$ tends to zero and $\max _{M_{s}}|u| \rightarrow 0$ as $s \rightarrow \infty$.

PROOF. This follows directly from Proposition 5.1, Theorem 5.1, and Lemma 5.1. 
REMARK 5.1. The assumption (5) implies that the beginning singularity is "crushing' and by Theorem 5.2 we have that the solution to (1) gives a foliation of this 'crushing' singularity.

\section{Existence for all times and the Friedmann models}

In this section we look at the condition (5) when the cosmological spacetime is a Robertson-Walker spacetime or a Friedmann model. A Robertson-Walker spacetime is a warped product $M(k, f)=I \times_{f} S$ (see for example [21, Chapter 7 and 12]), where $S$ is a connected, often three-dimensional Riemannian manifold of constant curvature $k=-1,0$ or 1 and $f>0$ is a smooth function on an open interval $I$ in the one-dimensional Minkowski space $\mathbb{R}_{1}^{1}$. To satisfy our definition of cosmological spacetime we also need that $S$ is compact. We talk about a Friedmann cosmological model, if $n=3$ and the scale function $f$ in a Robertson-Walker spacetime satisfies the Friedmann equation $f^{\prime 2}+k=A / f$, where $A$ is a constant depending only on mass. This is a model for $d u s t$ (noninteracting matter).

PROPOSITION 6.1. In a Robertson-Walker spacetime condition (5) takes the following form for the scale function

$$
\hat{\delta} f(t) \leq n f^{\prime}(t) t \leq \hat{C} f(t)
$$

for $\hat{\delta}, \hat{C}>0$.

PROOF. That the 'big bang' is at $t=0$, can always be achieved by rescaling time $(f(0)=0)$. General results about Robertson-Walker spacetimes [21, Chapter 12] show that $d / d t H_{S}=\left(\left|A_{S}\right|^{2}+\overline{\operatorname{Ric}}(T, T)\right)$ and since $H_{S}=n f^{\prime} / f$ the last two inequalities reduce to $(6)$.

PROPOSITION 6.2. The condition (5) is always satisfied in a Friedmann model.

PROOF. It is enough to show that (6) is always true. For this we look at the solutions to the Friedmann equation, assuming the 'big bang' is at $t=0$

(a) $\quad k=0: \quad f(t)=C t^{2 / 3} \quad$ with $4 C^{3}=9 A$;

(B) $\quad k=1: \quad t=\frac{1}{2} A(\theta-\sin \theta), \quad f=\frac{1}{2} A(1-\cos \theta) \quad \theta \in(0,2 \pi) ;$

( $\gamma) \quad k=-1: \quad t=\frac{1}{2} A(\sinh \eta-\eta), \quad f=\frac{1}{2} A(\cosh \eta-1) \quad \eta>0$. 
In the case $(\alpha)$ we get $3 f^{\prime} t / f=2$ so that (6) is true with $\hat{\delta}=\hat{C}=2$. For $(\beta)$ we get $3 f^{\prime}\left(u_{0}\right) u_{0} / f\left(u_{0}\right) \leq 3 f^{\prime} t / f \leq 2$ where $u_{0}=\min _{M_{0}} u$. This gives us $\hat{\delta}=3 f\left(u_{0}\right)^{\prime} u_{0} / f\left(u_{0}\right)$ and $\hat{C}=2$. When $k=-1$ we get $2 \leq 3 f^{\prime} t / f \leq 3$ so that the constants for $(\gamma)$ are $\hat{\delta}=2$ and $\hat{C}=3$.

REMARK 6.1. The qualitative behaviour near the singularity of $(\alpha),(\beta)$ and $(\gamma)$ is the same (see for example [13]).

From the above two propositions follows the existence for all times, which we formulate in the following theorem.

THEOREM 6.1. In a Robertson-Walker spacetime where the time function satisfies the estimate (6) with constants $\hat{\delta}, \hat{C}>0$, a solution to (1) exists for all times $s \geq 0$ and therefore Theorem 5.2 holds.

PROOF. This result follows direct from the above propositions.

\section{References}

[1] R. Bartnik, 'Existence of maximal surfaces in asymptotically flat spacetimes', Comm. Math. Phys. 94 (1984), 155-175.

[2] —, 'Remarks on cosmological spacetimes and constant mean curvature surfaces', Comm. Math. Phys. 117 (1988), 615-624.

[3] S.-Y. Cheng and S.-T. Yau, 'Maximal space-like hypersurfaces in the Lorentz-Minkowski spaces', Ann. of Math. 104 (1976), 405-419.

[4] Y. Choquet-Bruhat, 'Maximal submanifolds and submanifolds with constant mean extrinsic curvature of a Lorentzian manifold', Ann. Scuola Norm. Sup. Pisa 3 (1976), 361-376.

[5] K. Ecker and G. Huisken, 'Interior estimates for hypersurfaces moving by mean curvature', Invent. Math. 105 (1991), 547-569.

[6] _ - 'Parabolic methods for the construction of spacelike slices of prescribed mean curvature in cosmological spacetimes', Comm. Math. Phys. 35 (1991), 595-613.

[7] A. Fischer and J. Marsden, 'Topics in the dynamics of general relativity, in isolated gravitating systems in general relativity', Italian Phys. Soc. (1979), 322-395.

[8] C. Gerhardt, ' $H$-surfaces in Lorentzian manifolds', Comm. Math. Phys. 89 (1983), 523-553.

[9] _ . 'Flow of nonconvex hypersurfaces into spheres', J. Differential Geom. 32 (1990), 299-314.

[10] R. S. Hamilton, 'Three-manifolds with positive Ricci curvature', J. Differential Geom. 17 (1982), 255-306.

[11] — , 'Four-manifolds with positive curvature operator', J. Differential Geom. 24 (1986), 153179.

[12] - Lecture notes (Hawaii, 1989).

[13] S. W. Hawking and G. F. R. Ellis, The large scale structure of space-time, Cambridge Monographs Math. Phys. (Cambridge University Press, Cambridge, 1973).

[14] G. Huisken, 'Flow by mean curvature of convex surfaces into spheres', J. Differential Geom. 10 (1984), 237-266. 
[15] - 'Contracting convex hypersurfaces in Riemannian manifolds by their mean curvature', Invent. Math. 84 (1986), 463-480.

[16] _ , 'Deforming hypersurfaces of the sphere by their mean curvature', Math. Z. 195 (1987), 205-219.

[17] G. Huisken and T. Ilmanen, 'The Riemannian Penrose inequality', Internat. Math. Res. Notices 20 (1997), 1045-1058.

[18] N. V. Krylov, Nonlinear elliptic and parabolic equations of the second order (D. Reidel Publ. Co., Dordrecht, 1987).

[19] O. A. Ladyshenzkaya, V. A. Solonnikov and N. N. Ural'ceva, Linear and quasilinear equations of parabolic type, English translation: Transl. Math. Monographs, Vol. 23 (Amer. Math. Soc., Rhode Island, 1967).

[20] S. Nishikawa, 'On maximal space-like hypersurfaces in a Lorentzian manifold', Nagoya Math. J. 95 (1984), 117-124.

[21] B. O'Neill, Semi-Riemannian geometry with applications to relativity, Pure and Appl. Math. 103 (Academic Press, New York, 1983).

[22] R. Schoen, L. Simon and S.-T. Yau, 'Curvature estimates for minimal hypersurfaces', Acta Math. 134 (1975), 275-288.

[23] J. I. E. Urbas, 'On the expansion of starshaped hypersurfaces by symmetric functions of their principal curvatures', Math. Z. 205 (1990), 355-372.

Mathematisches Institut

Universität Tübingen

Auf der Morgenstelle 10

D-72076 Tübingen

Germany

e-mail: michael@moebius.mathematik.uni-tuebingen.de 